\title{
Set-valued G-Prešić operators on metric spaces endowed with a graph and fixed point theorems
}

\author{
Naseer Shahzad ${ }^{1 *}$ and Satish Shukla ${ }^{2}$
}

"Correspondence:

nshahzad@kau.edu.sa

${ }^{1}$ Department of Mathematics, King

Abdulaziz University, P.O. Box 80203,

Jeddah, 21859, Saudi Arabia

Full list of author information is

available at the end of the article

\begin{abstract}
In this paper, we consider the set-valued contractions defined on product spaces when the underlying space is a complete metric space endowed with a graph. Some fixed point results for the so-called set-valued G-Prešić operators are established. Our theorems extend and generalize some known results in product spaces of the recent literature. As an application of our main result, fixed point results for various types of set-valued contractions on product spaces are derived, and a sufficient condition for the existence of a weakly asymptotically stable and global attractor equilibrium point of a kth order nonlinear difference inclusion is established.
\end{abstract}

MSC: $47 \mathrm{H} 10 ; 54 \mathrm{H} 25 ; 39 \mathrm{~A} 11$

Keywords: set-valued G-Prešić operator; fixed point; graph; difference inclusion

\section{Introduction}

In 1965, Prešić $[1,2]$ extended the famous Banach contraction principle to the product spaces and obtained some convergence results for some particular sequences. Prešić proved the following theorem.

Theorem 1.1 (Prešić) Let $(X, d)$ be a complete metric space, $k$ be a positive integer and $T: X^{k} \rightarrow X$ be a mapping satisfying the following contractive type condition:

$$
d\left(T\left(x_{1}, x_{2}, \ldots, x_{k}\right), T\left(x_{2}, x_{3}, \ldots, x_{k+1}\right)\right) \leq \sum_{i=1}^{k} q_{i} d\left(x_{i}, x_{i+1}\right)
$$

for every $x_{1}, x_{2}, \ldots, x_{k}, x_{k+1} \in X$, where $q_{1}, q_{2}, \ldots, q_{k}$ are nonnegative constants such that $q_{1}+$ $q_{2}+\cdots+q_{k}<1$. Then there exists a unique point $x \in X$ such that $T(x, x, \ldots, x)=x$. Moreover, if $x_{1}, x_{2}, \ldots, x_{k}$ are arbitrary points in $X$ and for $n \in \mathbb{N}$,

$$
x_{n+k}=T\left(x_{n}, x_{n+1}, \ldots, x_{n+k-1}\right),
$$

then the sequence $\left\{x_{n}\right\}$ is convergent and $\lim x_{n}=T\left(\lim x_{n}, \lim x_{n}, \ldots, \lim x_{n}\right)$.

A point $x \in X$ such that $T(x, x, \ldots, x)=x$ is called a fixed point of $T$. A mapping $T$ satisfying condition (1.1) is called a Prešić type operator. Note that (1.2) represents a nonlinear difference equation of order $k$ and the fixed point of $T$ is an equilibrium point of

\section{然 Springer}

(c) 2015 Shahzad and Shukla; licensee Springer. This is an Open Access article distributed under the terms of the Creative Commons Attribution License (http://creativecommons.org/licenses/by/4.0), which permits unrestricted use, distribution, and reproduction in any medium, provided the original work is properly credited. 
(1.2) (see [3]). Therefore the Prešić's theorem ensures the existence and uniqueness of an equilibrium point of difference equation (1.2). Prešic type operators have applications in solving nonlinear difference equations, cyclic systems and in the study of convergence of sequences; for example, see [1-6]. This interesting result of Prešić has been further extended and generalized by several authors in different directions; see, for instance, [7-20].

Let $A$ be any nonempty subset of a metric space $(X, d)$. For $x \in X$, we define the distance between the point $x$ and the set $A$ by

$$
d(x, A)=\inf \{d(x, y): y \in A\} .
$$

Let $\mathrm{CB}(X)$ denote the set of all nonempty closed bounded subsets of $X$. For $A, B \in \mathrm{CB}(X)$, define

$$
\begin{aligned}
& \delta(A, B)=\sup \{d(x, B): x \in A\}, \\
& H(A, B)=\max \{\delta(A, B), \delta(B, A)\} .
\end{aligned}
$$

Then $H$ is a metric on $\mathrm{CB}(X)$ and is called Pompeiu-Hausdorff metric. The following remark is a consequence of the definition of Pompeiu-Hausdorff metric.

Remark 1.2 Let $A, B \in \mathrm{CB}(X)$ and $h \in(1, \infty)$ be given. Then, for $a \in A$, there exists $b \in B$ such that $d(a, b) \leq h H(A, B)$.

Nadler [21] extended the Banach contraction principle for the set-valued mappings, that is, for the mappings defined from the space $X$ into the set $\mathrm{CB}(X)$. Nadler [21] proved the following fixed point theorem.

Theorem 1.3 (Nadler) Let $(X, d)$ be a complete metric space, and let $T$ be a mapping from $X$ into $\mathrm{CB}(X)$ such that for all $x, y \in X$,

$$
H(T(x), T(y)) \leq \lambda d(x, y)
$$

where $\lambda \in[0,1)$. Then $T$ has a fixed point, that is, there exists a point $x \in X$ such that $x \in T x$.

Shukla et al. [22] unified the results of Prešić and Nadler and studied the fixed point results for set-valued Prešić type mappings. The results of Shukla et al. [22] are generalized in the recent papers $[13,15,18]$.

In 2004, Ran and Reurings [23] initiated the fixed point theory in complete metric spaces endowed with a partial order. Luong and Thuan [20] and Shukla and Radenović [16] considered the Prešić type mappings in partially ordered sets and proved the ordered version of Prešic theorem. These results generalize the result of Ran and Reurings [23] in product spaces.

In 2008, Jachymski [24] presented a nice unification of most of the previous results on a fixed point in metric spaces endowed with a graph. Very recently, Shukla and Shahzad [14] extended, generalized and unified the result of Jachymski [24], Prešić [1, 2], Luong and Thuan [20] by proving fixed point results for G-Prešić type operators in the spaces endowed with a graph. Related results can also be found in [25-32]. 
In this paper, we introduce the notion of set-valued G-Prešić operators on the product spaces when the underlying space is endowed with a graph and prove some fixed point results for these operators which extend the result of Shukla et al. [22] in spaces endowed with a graph. An example which shows that this extension is proper is given. Our results generalize and unify the results of Jachymski [24], Prešić [1, 2], Luong and Thuan [20], Shukla and Shahzad [14] and several other existing results in the literature. By applying our main results, we derive several fixed point results for various set-valued Prešić type operators. A sufficient condition for the existence of a weakly asymptotically stable and global attractor equilibrium point of a $k$ th order nonlinear difference inclusion is provided.

\section{Preliminaries}

In this section we recall some definitions and facts about the graphs which will be useful in the sequel.

Let $(X, d)$ be a metric space. Let $\Delta$ denote the diagonal of the Cartesian product $X \times X$. Consider a directed graph $G$ such that the set $V(G)$ of its vertices coincides with $X$, and the set $E(G)$ of its edges contains all loops, that is, $E(G) \supseteq \Delta$. We assume that $G$ has no parallel edges, so we can identify $G$ with the pair $(V(G), E(G))$. Moreover, we may treat $G$ as a weighted graph by assigning to each edge the distance between its vertices.

By $G^{-1}$ we denote the conversion of a graph $G$, that is, the graph obtained from $G$ by reversing the direction of edges. Thus we have

$$
E\left(G^{-1}\right)=\{(x, y) \in X \times X:(y, x) \in E(G)\} .
$$

The letter $\widetilde{G}$ denotes the undirected graph obtained from $G$ by ignoring the direction of edges. Actually, it will be more convenient for us to treat $\widetilde{G}$ as a directed graph for which the set of its edges is symmetric. Under this convention,

$$
E(\widetilde{G})=E(G) \cup E\left(G^{-1}\right) .
$$

Now we recall a few basic notions concerning connectivity of graphs (see [33]). If $x$ and $y$ are vertices in a graph $G$, then a path in $G$ from $x$ to $y$ of length $N \in \mathbb{N} \cup\{0\}$ is a sequence $\left\{x_{i}\right\}_{i=0}^{N}$ of $N+1$ vertices such that $x_{0}=x, x_{N}=y$ and $\left(x_{i-1}, x_{i}\right) \in E(G)$ for $i=1, \ldots, N$. A graph $G$ is called connected if there is a path between any two vertices of $G$. $G$ is weakly connected if $\widetilde{G}$ is connected. A sequence $\left\{x_{n}\right\}$ in $X$ is called a termwise connected sequence if $\left(x_{n}, x_{n+1}\right) \in E(G)$ for all $n \in \mathbb{N}$.

Throughout this paper, we assume that $X$ is a nonempty set, $G$ is a directed graph such that $V(G)=X$ and $E(G) \supseteq \Delta$. For the mapping $T: X^{k} \rightarrow \mathrm{CB}(X)$, a point $x \in X$ is called a fixed point of $T$ if $x \in T(x, \ldots, x)$. We denote the set of all fixed points of $T$ by $\operatorname{Fix}(T)$.

\section{Main results}

First we define the set-valued G-Prešić operators on the metric spaces endowed with a graph.

Definition 3.1 Let $(X, d)$ be a metric space, $k$ be a positive integer and $T: X^{k} \rightarrow \mathrm{CB}(X)$ be a mapping. Suppose, for every path $\left\{x_{i}\right\}_{i=1}^{k+1}$ of $k+1$ vertices in $G$, that the following conditions are satisfied: 
(GP1) There exist nonnegative constants $\alpha_{i}^{\prime}$ s such that $\sum_{i=1}^{k} \alpha_{i}<1$ and

$$
H\left(T\left(x_{1}, x_{2}, \ldots, x_{k}\right), T\left(x_{2}, x_{3}, \ldots, x_{k+1}\right)\right) \leq \sum_{i=1}^{k} \alpha_{i} d\left(x_{i}, x_{i+1}\right) .
$$

(GP2) If $x_{k+1} \in T\left(x_{1}, x_{2}, \ldots, x_{k}\right)$ and $x_{k+2} \in T\left(x_{2}, x_{3}, \ldots, x_{k+1}\right)$ are such that $d\left(x_{k+1}, x_{k+2}\right)<\max \left\{d\left(x_{i}, x_{i+1}\right): i=1,2, \ldots, k\right\}$, then $\left(x_{k+1}, x_{k+2}\right) \in E(G)$.

Then the mapping $T$ is called a set-valued G-Prešić operator.

Remark 3.2 For $E(G)=X \times X$, a set-valued G-Prešić operator reduces into a set-valued Prešić type contraction (see Shukla et al. [22]).

Definition 3.3 Let $(X, d)$ be a metric space, $k$ be a positive integer and $T: X^{k} \rightarrow \mathrm{CB}(X)$ be a mapping. Define a mapping $\mathcal{T}: X \rightarrow \mathrm{CB}(X)$ by $\mathcal{T}(x)=T(x, x, \ldots, x)$ for all $x \in X$. Then the mapping $\mathcal{T}$ is called the associate operator of $T$.

The following remark will be useful in proving some consequences of our main result.

Remark 3.4 Let the mapping $T$ satisfy (GP1). If $(x, y) \in E(\widetilde{G})$ then

$$
H(\mathcal{T}(x), \mathcal{T}(y)) \leq\left[\sum_{i=1}^{k} \alpha_{i}\right] d(x, y) .
$$

Proof Let $(x, y) \in E(\widetilde{G})=E(G) \cup E\left(G^{-1}\right)$. If $(x, y) \in E(G)$ then, since $E(G) \supseteq \Delta$, by (GP1) we have

$$
\begin{aligned}
H(\mathcal{T}(x), \mathcal{T}(y)) \leq & H(T(x, \ldots, x), T(x, \ldots, x, y)) \\
& +H(T(x, \ldots, x, y), T(x, \ldots, x, y, y))+\cdots \\
& +H(T(x, y, \ldots, y), T(y, \ldots, y)) \\
\leq & \alpha_{k} d(x, y)+\alpha_{k-1} d(x, y)+\cdots+\alpha_{1} d(x, y) \\
= & {\left[\sum_{i=1}^{k} \alpha_{i}\right] d(x, y) . }
\end{aligned}
$$

Similarly, if $(x, y) \in E\left(G^{-1}\right)$ we obtain the same result.

Now we prove an existence theorem for a set-valued G-Prešić operator.

Theorem 3.5 Let $(X, d)$ be a complete metric space, $k$ be a positive integer and $T: X^{k} \rightarrow$ $\mathrm{CB}(X)$ be a set-valued G-Prešić operator. Suppose that the following conditions hold:

(a) There exists a path $\left\{x_{i}\right\}_{i=1}^{k+1}$ of $k+1$ vertices in $G$ such that $x_{k+1} \in T\left(x_{1}, x_{2}, \ldots, x_{k}\right)$.

(b) For any termwise connected sequence $\left\{x_{n}\right\}$ in $X$ if $x_{n} \rightarrow x$ and

$x_{n+k} \in T\left(x_{n}, x_{n+1}, \ldots, x_{n+k-1}\right)$ for all $n \in \mathbb{N}$, then there exists a subsequence $\left\{x_{n_{j}}\right\}$ such that $\left(x_{n_{j}}, x\right) \in E(G)$ for all $j \in \mathbb{N}$.

Then $T$ has a fixed point in $X$. Moreover, there exists a termwise connected sequence $\left\{x_{n}\right\}$ in $X$ such that $x_{n+k} \in T\left(x_{n}, x_{n+1}, \ldots, x_{n+k-1}\right)$ for all $n \in \mathbb{N}$ and $\left\{x_{n}\right\}$ converges to a fixed point of $T$. 
Proof Let $\lambda=\sum_{i=1}^{k} \alpha_{i}<1$. Suppose that there is a path $\left\{x_{i}\right\}_{i=1}^{k+1}$ of $k+1$ vertices in $G$ such that $x_{k+1} \in T\left(x_{1}, x_{2}, \ldots, x_{k}\right)$. Then, since $T\left(x_{2}, x_{3}, \ldots, x_{k+1}\right) \in \mathrm{CB}(X)$ and $\lambda<1$, by Remark 1.2 there exists $x_{k+2} \in T\left(x_{2}, x_{3}, \ldots, x_{k+1}\right)$ such that

$$
d\left(x_{k+1}, x_{k+2}\right) \leq \frac{1}{\sqrt{\lambda}} H\left(T\left(x_{1}, x_{2}, \ldots, x_{k}\right), T\left(x_{2}, x_{3}, \ldots, x_{k+1}\right)\right)
$$

As $\left\{x_{i}\right\}_{i=1}^{k+1}$ is a path of $k+1$ vertices in $G$ and $T$ is a set-valued $G$-Prešić operator, it follows from (GP1) and the above inequality that

$$
\begin{aligned}
d\left(x_{k+1}, x_{k+2}\right) & \leq \frac{1}{\sqrt{\lambda}} \sum_{i=1}^{k} \alpha_{i} d\left(x_{i}, x_{i+1}\right) \\
& \leq \frac{1}{\sqrt{\lambda}}\left(\sum_{i=1}^{k} \alpha_{i}\right) \max \left\{d\left(x_{j}, x_{j+1}\right): j=1,2, \ldots, k\right\} \\
& =\sqrt{\lambda} \max \left\{d\left(x_{j}, x_{j+1}\right): j=1,2, \ldots, k\right\} \\
& <\max \left\{d\left(x_{j}, x_{j+1}\right): j=1,2, \ldots, k\right\} .
\end{aligned}
$$

By (GP2) and the above inequality, we have $\left(x_{k+1}, x_{k+2}\right) \in E(G)$. Thus, $\left\{x_{i}\right\}_{i=2}^{k+2}$ is a path of $k+1$ vertices in $G$. Again, since $T\left(x_{3}, x_{4}, \ldots, x_{k+3}\right) \in \mathrm{CB}(X)$ by Remark 1.2 , there exists $x_{k+3} \in T\left(x_{3}, x_{4}, \ldots, x_{k+2}\right)$ such that

$$
d\left(x_{k+2}, x_{k+3}\right) \leq \frac{1}{\sqrt{\lambda}} H\left(T\left(x_{2}, x_{3}, \ldots, x_{k+1}\right), T\left(x_{3}, x_{4}, \ldots, x_{k+2}\right)\right)
$$

and by (GP1) we have

$$
\begin{aligned}
d\left(x_{k+2}, x_{k+3}\right) & \leq \frac{1}{\sqrt{\lambda}} \sum_{i=1}^{k} \alpha_{i} d\left(x_{i+1}, x_{i+2}\right) \\
& \leq \frac{1}{\sqrt{\lambda}}\left(\sum_{i=1}^{k} \alpha_{i}\right) \max \left\{d\left(x_{j+1}, x_{j+2}\right): j=1,2, \ldots, k\right\} \\
& =\sqrt{\lambda} \max \left\{d\left(x_{j+1}, x_{j+2}\right): j=1,2, \ldots, k\right\} \\
& <\max \left\{d\left(x_{j+1}, x_{j+2}\right): j=1,2, \ldots, k\right\} .
\end{aligned}
$$

By (GP2) and the above inequality, we have $\left(x_{k+2}, x_{k+3}\right) \in E(G)$. Proceeding in this manner, we obtain a sequence $\left\{x_{n}\right\}$ such that $\left(x_{n}, x_{n+1}\right) \in E(G), x_{n+k} \in T\left(x_{n}, x_{n+1}, \ldots, x_{n+k-1}\right)$ for all $n \in \mathbb{N}$ and

$$
d\left(x_{n+k}, x_{n+k+1}\right) \leq \sqrt{\lambda} \max \left\{d\left(x_{j+n-1}, x_{j+n}\right): j=1,2, \ldots, k\right\} \quad \text { for all } n \in \mathbb{N}
$$

We shall show that the sequence $\left\{x_{n}\right\}$ is a Cauchy sequence.

Let

$$
\mu=\max \left\{\frac{d\left(x_{i}, x_{i+1}\right)}{\vartheta^{i}}: i=1,2, \ldots, k\right\}
$$


where $\vartheta=\lambda^{1 / 2 k}$. By the method of mathematical induction we shall prove that

$$
d\left(x_{n}, x_{n+1}\right) \leq \mu \vartheta^{n} \quad \text { for all } n \in \mathbb{N} \text {. }
$$

Then, by the definition of $\mu$, it is clear that inequality (3.2) is true for $n=1,2, \ldots, k$. Let the $k$ inequalities $d\left(x_{n}, x_{n+1}\right) \leq \mu \vartheta^{n}, d\left(x_{n+1}, x_{n+2}\right) \leq \mu \vartheta^{n+1}, \ldots, d\left(x_{n+k-1}, x_{n+k}\right) \leq \mu \vartheta^{n+k-1}$ be the induction hypothesis. For every $n \in \mathbb{N}$, it follows from (3.1) that

$$
\begin{aligned}
d\left(x_{n+k}, x_{n+k+1}\right) & \leq \sqrt{\lambda} \max \left\{d\left(x_{j+n-1}, x_{j+n}\right): j=1,2, \ldots, k\right\} \\
& \leq \sqrt{\lambda} \max \left\{\mu \vartheta^{j+n-1}: j=1,2, \ldots, k\right\} \\
& \leq \sqrt{\lambda} \mu \vartheta^{n} \quad\left(\text { as } \vartheta=\lambda^{1 / 2 k}<1\right) \\
& =\mu \vartheta^{n+k} .
\end{aligned}
$$

Thus, the inductive proof of (3.2) is complete. Now, for $n, m \in \mathbb{N}, m>n$, using (3.2) we obtain

$$
\begin{aligned}
d\left(x_{n}, x_{m}\right) & \leq d\left(x_{n}, x_{n+1}\right)+d\left(x_{n+1}, x_{n+2}\right)+\cdots+d\left(x_{m-1}, x_{m}\right) \\
& \leq \mu \vartheta^{n}+\mu \vartheta^{n+1}+\cdots+\mu \vartheta^{m-1} \\
& \leq \mu \vartheta^{n}\left[1+\vartheta+\vartheta^{2}+\cdots\right] \\
& =\frac{\mu \vartheta^{n}}{1-\vartheta} .
\end{aligned}
$$

Since $\vartheta=\lambda^{1 / 2 k}<1$, it follows from the above inequality that

$$
\lim _{n, m \rightarrow \infty} d\left(x_{n}, x_{m}\right)=0
$$

Therefore, $\left\{x_{n}\right\}$ is a Cauchy sequence in $X$. By completeness of $X$, there exists $u \in X$ such that

$$
\lim _{n \rightarrow \infty} d\left(x_{n}, u\right)=0 .
$$

We shall show that $u$ is a fixed point of $T$. By (b) there exists a subsequence $\left\{x_{n_{j}}\right\}$ such that $\left(x_{n_{j}}, u\right) \in E(G)$ for all $j \in \mathbb{N}$. Since for each $n \in \mathbb{N}$ we have $\left(x_{n}, x_{n+1}\right) \in E(G)$ and $x_{n+k} \in$ $T\left(x_{n}, x_{n+1}, \ldots, x_{n+k-1}\right)$, therefore, for any $j \in \mathbb{N}$, we have

$$
\begin{aligned}
d(u, T(u, \ldots, u)) \leq & d\left(u, x_{n_{j}+k}\right)+d\left(x_{n_{j}+k}, T(u, \ldots, u)\right) \\
\leq & d\left(u, x_{n_{j}+k}\right)+H\left(T\left(x_{n_{j}}, x_{n_{j}+1}, \ldots, x_{n_{j}+k-1}\right), T(u, \ldots, u)\right) \\
\leq & d\left(u, x_{n_{j}+k}\right)+H\left(T\left(x_{n_{j}}, x_{n_{j}+1}, \ldots, x_{n_{j}+k-1}\right), T\left(x_{n_{j}+1}, \ldots, x_{n_{j}+k-1}, u\right)\right) \\
& +H\left(T\left(x_{n_{j}+1}, \ldots, x_{n_{j}+k-1}, u\right), T\left(x_{n_{j}+2}, \ldots, x_{n_{j}+k-1}, u, u\right)\right) \\
& +\cdots+H\left(T\left(x_{n_{j}+k-1}, u, \ldots, u\right), T(u, \ldots, u)\right) \\
\leq & d\left(u, x_{n_{j}+k}\right)+\left\{\alpha_{1} d\left(x_{n_{j}}, x_{n_{j}+1}\right)+\cdots+\alpha_{k-1} d\left(x_{n_{j}+k-2}, x_{n_{j}+k-1}\right)\right. \\
& \left.+\alpha_{k} d\left(x_{n_{j}+k-1}, u\right)\right\}+\left\{\alpha_{1} d\left(x_{n_{j}+1}, x_{n_{j}+2}\right)+\cdots+\alpha_{k-2} d\left(x_{n_{j}+k-2}, x_{n_{j}+k-1}\right)\right. \\
& \left.+\alpha_{k-1} d\left(x_{n_{j}+k-1}, u\right)\right\}+\cdots+\alpha_{1} d\left(x_{n_{j}+k-1}, u\right) .
\end{aligned}
$$


Letting $j \rightarrow \infty$ in the above inequality, we obtain $d(u, T(u, \ldots, u))=0$, that is, $u \in$ $T(u, \ldots, u)$. Thus, $u$ is a fixed point of $T$.

The next example illustrates the above theorem; also, it shows the case when similar results from Shukla et al. [22] are not applicable but the new results are applicable.

Example 3.6 Let $\mathbb{N}_{0}=\mathbb{N} \cup\{0\}, X=\left\{0, \frac{1}{2^{n}}: n \in \mathbb{N}_{0}\right\}$ and define a graph $G$ by $V(G)=X$, $E(G)=\Delta \cup\left\{\left(\frac{1}{2^{n}}, \frac{1}{2^{n+1}}\right),\left(\frac{1}{2^{n}}, 0\right): n \in \mathbb{N}\right\}$. Then $(X, d)$ is a complete metric space. For $k=2$, define a mapping $T: X^{2} \rightarrow \mathrm{CB}(X)$ by

$$
T(x, y)= \begin{cases}\{x\} & \text { if } x=y \in\{0,1\} \\ \left\{\frac{1}{2^{n+3}}, \frac{1}{2^{n+4}}\right\} & \text { if } x=\frac{1}{2^{n}}, y=\frac{1}{2^{n+1}}, n \in \mathbb{N}_{0} \\ \{0\}, & \text { otherwise. }\end{cases}
$$

Then $T$ is a set-valued G-Prešić operator with $\alpha_{1}=\alpha_{2}=\frac{1}{4}$. All the conditions of Theorem 3.5 are satisfied and $\operatorname{Fix}(T)=\{0,1\}$. On the other hand, $T$ is not a set-valued Prešić type contraction in the sense of Shukla et al. [22]. Indeed, for $x_{1}=x_{2}=1, x_{3}=0$, we have

$$
H\left(T\left(x_{1}, x_{2}\right), T\left(x_{2}, x_{3}\right)\right)=H(\{1\},\{0\})=1
$$

and $d\left(x_{1}, x_{2}\right)=0, d\left(x_{2}, x_{3}\right)=1$. Therefore, we cannot find nonnegative constants $\alpha_{1}, \alpha_{2}$ such that $\alpha_{1}+\alpha_{2}<1$ and

$$
H\left(T\left(x_{1}, x_{2}\right), T\left(x_{2}, x_{3}\right)\right) \leq \alpha_{1} d\left(x_{1}, x_{2}\right)+\alpha_{2} d\left(x_{2}, x_{3}\right) .
$$

If we define the graph $G$ by $V(G)=X$ and $E(G)=X \times X$ in Theorem 3.5, then $E(G) \supseteq \Delta$ and $G$ has no parallel edges, and so we obtain the following corollary, which is an existence theorem for the set-valued Prešić type contraction (for the related definitions and results, see [22]).

Corollary 3.7 Let $(X, d)$ be a complete metric space, $k$ be a positive integer and $T: X^{k} \rightarrow$ $\mathrm{CB}(X)$ be a set-valued Prešić type contraction, that is, the following condition holds:

$$
H\left(T\left(x_{1}, x_{2}, \ldots, x_{k}\right), T\left(x_{2}, x_{3}, \ldots, x_{k+1}\right)\right) \leq \sum_{i=1}^{k} \alpha_{i} d\left(x_{i}, x_{i+1}\right)
$$

for all $x_{1}, x_{2}, \ldots, x_{k+1} \in X$, where $\alpha_{i}$ are nonnegative constants such that $\sum_{i=1}^{k} \alpha_{i}<1$. Then $T$ has a fixed point in $X$. Moreover, for arbitrary $x_{1}, x_{2}, \ldots, x_{k} \in X$, there exists a sequence $\left\{x_{n}\right\}$ in $X$ such that $x_{n+k} \in T\left(x_{n}, x_{n+1}, \ldots, x_{n+k-1}\right)$ for all $n \in \mathbb{N}$ and $\left\{x_{n}\right\}$ converges to a fixed point of $T$.

\section{Applications to some fixed point results in product spaces}

In this section, we apply the results of the previous section and establish some fixed point results for Prešić type operators in various settings.

First, we give the following theorem for set-valued Prešić type operators in $\varepsilon$-chainable spaces (for related definitions, see [34]) which is new even for a single-valued case. 
Theorem 4.1 Let $(X, d)$ be a complete $\varepsilon$-chainable space, $k$ be a positive integer and $T: X^{k} \rightarrow \mathrm{CB}(X)$ be a mapping. Suppose that the following condition holds:

$$
H\left(T\left(x_{1}, x_{2}, \ldots, x_{k}\right), T\left(x_{2}, x_{3}, \ldots, x_{k+1}\right)\right) \leq \sum_{i=1}^{k} \alpha_{i} d\left(x_{i}, x_{i+1}\right)
$$

for all $x_{1}, x_{2}, \ldots, x_{k}, x_{k+1} \in X$ with $\max \left\{d\left(x_{i}, x_{i+1}\right): 1 \leq i \leq k\right\}<\varepsilon$, where $\alpha_{i}$ 's are nonnegative constants such that $\sum_{i=1}^{k} \alpha_{i}<1$. Suppose that there exist points $x_{1}, \ldots, x_{k}, x_{k+1}$ such that $\max \left\{d\left(x_{i}, x_{i+1}\right): 1 \leq i \leq k\right\}<\varepsilon$ and $x_{k+1} \in T\left(x_{1}, x_{2}, \ldots, x_{k}\right)$. Then $T$ has a fixed point in X. Moreover, there exists a sequence $\left\{x_{n}\right\}$ in $X$ such that $x_{n+k} \in T\left(x_{n}, x_{n+1}, \ldots, x_{n+k-1}\right)$, $d\left(x_{n}, x_{n+1}\right)<\varepsilon$ for all $n \in \mathbb{N}$ and $\left\{x_{n}\right\}$ converges to a fixed point of $T$.

Proof Consider the graph $G$ with $V(G)=X$ and

$$
E(G)=\{(x, y) \in X \times X: d(x, y)<\varepsilon\} .
$$

Obviously, $E(G) \supseteq \Delta$ and $G$ has no parallel edges. By some easy calculations one can see that $T$ is a set-valued G-Prešić operator. By assumption, condition (a) of Theorem 3.5 is satisfied. Also, if $\left\{x_{n}\right\}$ is a sequence in $X$ such that $x_{n} \rightarrow x \in X$, then there exists $n_{0} \in \mathbb{N}$ such that $d\left(x_{n}, x\right)<\varepsilon$ for all $n>n_{0}$. Therefore, we can construct a subsequence $\left\{x_{n_{j}}\right\}$ such that $\left(x_{n_{j}}, x\right) \in E(G)$ for all $j \in \mathbb{N}$. Thus, all the conditions of Theorem 3.5 are satisfied, and so the result follows.

The following corollary is an extension and generalization of the result of Prešić $[1,2]$ on $\varepsilon$-chainable spaces, and it extends the result of Edelstein [34] in product spaces.

Corollary 4.2 Let $(X, d)$ be a complete $\varepsilon$-chainable space, $k$ be a positive integer and $T: X^{k} \rightarrow X$ be a mapping. Suppose that the following condition holds:

$$
d\left(T\left(x_{1}, x_{2}, \ldots, x_{k}\right), T\left(x_{2}, x_{3}, \ldots, x_{k+1}\right)\right) \leq \sum_{i=1}^{k} \alpha_{i} d\left(x_{i}, x_{i+1}\right)
$$

for all $x_{1}, x_{2}, \ldots, x_{k}, x_{k+1} \in X$ with $\max \left\{d\left(x_{i}, x_{i+1}\right): 1 \leq i \leq k\right\}<\varepsilon$, where $\alpha_{i}$ 's are nonnegative constants such that $\sum_{i=1}^{k} \alpha_{i}<1$. Suppose that there exist points $x_{1}, \ldots, x_{k}, x_{k+1}$ such that $\max \left\{d\left(x_{i}, x_{i+1}\right): 1 \leq i \leq k\right\}<\varepsilon$ and $x_{k+1}=T\left(x_{1}, x_{2}, \ldots, x_{k}\right)$. Then $T$ has a unique fixed point in $X$. Moreover, there exists a sequence $\left\{x_{n}\right\}$ in $X$ such that $x_{n+k}=T\left(x_{n}, x_{n+1}, \ldots, x_{n+k-1}\right)$, $d\left(x_{n}, x_{n+1}\right)<\varepsilon$ for all $n \in \mathbb{N}$ and $\left\{x_{n}\right\}$ converges to the fixed point of $T$.

Proof Consider the graph $G$ with $V(G)=X$ and

$$
E(G)=\{(x, y) \in X \times X: d(x, y)<\varepsilon\} .
$$

Obviously, $E(G) \supseteq \Delta$ and $G$ has no parallel edges. The existence of a fixed point is obvious. For uniqueness, let $u, v \in \operatorname{Fix}(T), u \neq v$, then by $\varepsilon$-chainability of $(X, d)$, there exists a sequence $\left\{x_{i}\right\}_{i=0}^{N}, x_{0}=u, x_{N}=v$ and $d\left(x_{i-1}, x_{i}\right)<\varepsilon$ for $i=1,2, \ldots, N$. Define $\mathcal{T}: X \rightarrow X$, the associate operator of $T$, by $\mathcal{T}(x)=T(x, x, \ldots, x)$ for all $x \in X$. Then it is clear that $u \in X$ 
is a fixed point of $T$ if and only if it is a fixed point of $\mathcal{T}$. Note that, for any $x, y \in X$ with $d(x, y)<\varepsilon$, by Remark 3.4 we have

$$
d(\mathcal{T}(x), \mathcal{T}(y))=d(T(x, \ldots, x), T(y, \ldots, y)) \leq\left[\sum_{i=1}^{k} \alpha_{i}\right] d(x, y)<\varepsilon .
$$

Therefore, by repetition of this process, for all $x, y \in X$ with $d(x, y)<\varepsilon$, we have

$$
d\left(\mathcal{T}^{m}(x), \mathcal{T}^{m}(y)\right) \leq\left[\sum_{i=1}^{k} \alpha_{i}\right]^{m} d(x, y) \quad \text { for all } m \in \mathbb{N}
$$

Now,

$$
d(u, v)=\sum_{i=1}^{N} d\left(\mathcal{T}^{m}\left(x_{i-1}\right), \mathcal{T}^{m}\left(x_{i}\right)\right) \leq\left[\sum_{i=1}^{k} \alpha_{i}\right]^{m} \sum_{i=1}^{N} d\left(x_{i-1}, x_{i}\right) .
$$

As $\sum_{i=1}^{k} \alpha_{i}<1$, letting $m \rightarrow \infty$ we obtain $d(u, v)=0$, i.e., $u=v$. This contradiction shows that the fixed point of $T$ is unique.

Let $(X, \sqsubseteq)$ be a partially ordered set such that $d$ is a metric on $X$, then the triple $(X, \sqsubseteq, d)$ is called an ordered metric space. A subset $A \subseteq X$ is called well-ordered if, for all $x, y \in X$, either $x \sqsubseteq y$ or $y \sqsubseteq x$. A sequence $\left\{x_{i}\right\}_{i=1}^{n}$ is called nondecreasing with respect to $\sqsubseteq$ if $x_{i} \sqsubseteq$ $x_{i+1}, i=1,2, \ldots, n-1$. Next, we define set-valued ordered Prešić operators on an ordered metric space.

Definition 4.3 Let $(X, \sqsubseteq, d)$ be an ordered metric space, $k$ be a positive integer and $T: X^{k} \rightarrow \mathrm{CB}(X)$ be a mapping. Then the mapping $T$ is called a set-valued ordered Prešić operator if:

(OP1) for a nondecreasing sequence $\left\{x_{i}\right\}_{i=1}^{k+1}$ with respect to $\sqsubseteq$, we have

$$
H\left(T\left(x_{1}, x_{2}, \ldots, x_{k}\right), T\left(x_{2}, x_{3}, \ldots, x_{k+1}\right)\right) \leq \sum_{i=1}^{k} \alpha_{i} d\left(x_{i}, x_{i+1}\right),
$$

where $\alpha_{i}$ 's are nonnegative constants such that $\sum_{i=1}^{k} \alpha_{i}<1$;

(OP2) if $\left\{x_{i}\right\}_{i=1}^{k+1}$ is a nondecreasing sequence with respect to $\sqsubseteq, x_{k+1} \in T\left(x_{1}, \ldots, x_{k}\right)$ and $x_{k+2} \in T\left(x_{2}, \ldots, x_{k+1}\right)$ are such that $d\left(x_{k+1}, x_{k+2}\right)<\max \left\{d\left(x_{i}, x_{i+1}\right): i=1,2, \ldots, k\right\}$, then $x_{k+1} \sqsubseteq x_{k+2}$.

The following theorem is a fixed point result for a set-valued ordered Prešić operator on an ordered metric space and it extends the results of Malhotra et al. [9] and Luong and Thuan [20] for set-valued mappings.

Theorem 4.4 Let $(X, \sqsubseteq, d)$ be a complete, ordered metric space, $k$ be a positive integer and $T: X^{k} \rightarrow \mathrm{CB}(X)$ be a set-valued ordered Prešić operator. Suppose that the following conditions hold:

(a) There exists a nondecreasing sequence $\left\{x_{i}\right\}_{i=1}^{k+1}$ such that $x_{k+1} \in T\left(x_{1}, \ldots, x_{k}\right)$. 
(b) For any sequence $\left\{x_{n}\right\}$ in $X$, if $x_{n} \rightarrow x, x_{n} \sqsubseteq x_{n+1}$ and $x_{n+k} \in T\left(x_{n}, x_{n+1}, \ldots, x_{n+k-1}\right)$ for all $n \in \mathbb{N}$, then there exists a subsequence $\left\{x_{n_{j}}\right\}$ such that $x_{n_{j}} \sqsubseteq x$ for all $j \in \mathbb{N}$.

Then $T$ has a fixed point in X. Moreover, there exists a sequence $\left\{x_{n}\right\}$ in $X$ such that $x_{n+k} \in$ $T\left(x_{n}, x_{n+1}, \ldots, x_{n+k-1}\right), x_{n} \sqsubseteq x_{n+1}$ for all $n \in \mathbb{N}$, and $\left\{x_{n}\right\}$ converges to a fixed point of $T$.

Proof Consider the graph $G$ with $V(G)=X$ and

$$
E(G)=\{(x, y) \in X \times X: x \sqsubseteq y\} .
$$

Obviously, $E(G) \supseteq \Delta$ and $G$ has no parallel edges. Now it is easy to see that $T$ is a set-valued G-Prešić operator. Conditions (a) and (b) of Theorem 3.5 are satisfied trivially. Therefore, by Theorem 3.5, $T$ has a fixed point in $X$.

\section{Weak stability and weak asymptotic stability}

In this section, we consider the weak stability and attractivity of the equilibrium point of a $k$ th order nonlinear difference inclusion.

In the further discussion, we assume that $\mathfrak{B}$ is a Banach space with the norm $\|\cdot\|$ and $A$, a nonempty subset of $\mathfrak{B}$.

Let $T: A^{k} \rightarrow 2^{A}$ be a set-valued mapping with nonempty values at arbitrary points $x_{1}, x_{2}, \ldots, x_{k} \in A$, and consider the $k$ th order nonlinear difference inclusion on $A$ :

$$
x_{n+k} \in T\left(x_{n}, x_{n+1}, \ldots, x_{n+k-1}\right), \quad n=1,2, \ldots
$$

Solution to (5.1) is the functions $\psi: \mathbb{N} \rightarrow A$ such that for every $n \in \mathbb{N}, \psi(n+k)=\psi_{n+k} \in$ $T\left(\psi_{n}, \psi_{n+1}, \ldots, \psi_{n+k-1}\right)$. A point $u \in A$ is called an equilibrium point of $k$ th order nonlinear difference inclusion (5.1) if $u \in T(u, u, \ldots, u)$, that is, $u$ is a fixed point of the mapping $T$. An equilibrium point $u \in A$ of (5.1) is called weakly stable if, given $\varepsilon>0$, there exists $\delta>0$ such that for at least one solution of (5.1) with initial values $x_{1}, x_{2}, \ldots, x_{k}$ and $\left\|x_{1}-u\right\|+$ $\left\|x_{2}-u\right\|+\cdots+\left\|x_{k}-u\right\|<\delta$ implies $\left\|x_{n}-u\right\|<\varepsilon$ for all $n \in \mathbb{N}$. The equilibrium point $u \in A$ is called weakly asymptotically stable if, in addition to being weakly stable, $\lim _{n \rightarrow \infty} x_{n}=u$. The equilibrium point $u \in A$ is called a global attractor if, for arbitrary $x_{1}, x_{2}, \ldots, x_{k} \in A$, we have $\lim _{n \rightarrow \infty} x_{n}=u$.

Theorem 5.1 Let $A$ be a closed subset of the Banach space $\mathfrak{B}$ and $T: A^{k} \rightarrow \mathrm{CB}(A)$ be a set-valued Prešic type contraction, then for every set of initial conditions $x_{1}, x_{2}, \ldots, x_{k} \in A$ the difference inclusion (5.1) has an equilibrium point $u \in A$. Furthermore, the equilibrium point $u$ is weakly asymptotically stable and a global attractor.

Proof Define the graph $G$ by $V(G)=A$ and $E(G)=A \times A$. Then, by Corollary 3.7, $T$ has a fixed point in $A$, and this fixed point is an equilibrium point of the $k$ th order nonlinear difference inclusion (5.1). Furthermore, since $E(G)=A \times A$, for arbitrary $x_{1}, x_{2}, \ldots, x_{k} \in$ $A$, the sequence $\left\{x_{n}\right\}$ defined by $x_{n+k} \in T\left(x_{n}, x_{n+1}, \ldots, x_{n+k-1}\right)$ for all $n \in \mathbb{N}$ converges to $u$, therefore, $u$ is weakly asymptotically stable and a global attractor. 
Authors' contributions

All authors contributed equally to the writing of this paper. All authors read and approved the final manuscript.

\section{Author details}

'Department of Mathematics, King Abdulaziz University, P.O. Box 80203, Jeddah, 21859, Saudi Arabia. ${ }^{2}$ Department of Applied Mathematics, Shri Vaishnav Institute of Technology \& Science, Gram Baroli Sanwer Road, Indore, MP 453331, India.

\section{Acknowledgements}

This article was funded by the Deanship of Scientific Research (DSR), King Abdulaziz University, Jeddah. The first author acknowledges with thanks DSR for financial support. The authors would like to thank the reviewers for their valuable suggestions.

Received: 16 September 2014 Accepted: 8 January 2015 Published online: 13 February 2015

\section{References}

1. Prešić, SB: Sur la convergence des suites. C. R. Acad. Sci. Paris 260, 3828-3830 (1965)

2. Prešić, SB: Sur une classe d'inéquations aux différences finite et sur la convergence de certaines suites. Publ. Inst. Math. (Belgr.) 5(19), 75-78 (1965)

3. Shukla, S, Radenović, S: Some generalizations of Prešić type mappings and applications. An. Ştiinţ. Univ. 'Al.I. Cuza' laşi, Mat. (2014, accepted)

4. Khan, MS, Berzig, M, Samet, B: Some convergence results for iterative sequences of Prešić type and applications. Adv. Differ. Equ. (2012). doi:10.1186/1687-1847-2012-38

5. Chen, YZ: A Prešić type contractive condition and its applications. Nonlinear Anal. 71(12), 2012-2017 (2009)

6. Berinde, V, Pǎcurar, M: Two elementary applications of some Prešić type fixed point theorems. Creative Math. Inform. 20(1), 32-42 (2011)

7. Ćirić, LB, Prešić, SB: On Prešić type generalisation of Banach contraction principle. Acta Math. Univ. Comen. LXXVI(2), 143-147 (2007)

8. George, R, Reshma, KP, Rajagopalan, R: A generalised fixed point theorem of Prešić type in cone metric spaces and application to Morkov process. Fixed Point Theory Appl. 2011, 85 (2011). doi:10.1186/1687-1812-2011-85

9. Malhotra, SK, Shukla, S, Sen, R: Some coincidence and common fixed point theorems for Prešić-Reich type mappings in cone metric spaces. Rend. Semin. Mat. (Torino) 70(3), 247-260 (2012)

10. Păcurar, M: A multi-step iterative method for approximating common fixed points of Prešić-Rus type operators on metric spaces. Stud. Univ. Babeş-Bolyai, Math. LV(1), 149-162 (2010)

11. Pǎcurar, M: Approximating common fixed points of Prešić-Kannan type operators by a multi-step iterative method. An. Ştiinţ. Univ. 'Ovidius' Constanţa 17(1), 153-168 (2009)

12. Pǎcurar, M: Common fixed points for almost Prešić type operators. Carpath. J. Math. 28(1), 117-126 (2012)

13. Shukla, S: Some stability results and Assad-Kirk type fixed point theorems for set-valued Prešić type mappings. J. Nonlinear Convex Anal. 16(3), 509-520 (2015)

14. Shukla, S, Shahzad, N: G-Prešić operators on metric spaces endowed with a graph and fixed point theorems. Fixed Point Theory Appl. 2014, 127 (2014)

15. Shukla, S, Sen, R: Set-valued Prešić-Reich type mappings in metric spaces. Rev. R. Acad. Cienc. Exactas Fís. Nat., Ser. A Mat. (2012). doi:10.1007/s13398-012-0114-2

16. Shukla, S, Radenović, S: A generalization of Prešić type mappings in 0-complete ordered partial metric spaces. Chin. J. Math. 2013, Article ID 859531 (2013)

17. Shukla, S, Radojević, S, Veljković, ZA, Radenović, S: Some coincidence and common fixed point theorems for ordered Prešić-Reich type contractions. J. Inequal. Appl. 2013, 520 (2013). doi:10.1186/1029-242X-2013-520

18. Shukla, S: Set-valued Prešić-Ćirić type contraction in 0-complete partial metric spaces. Mat. Vesn. 66(2), 178-189 (2014)

19. Shukla, S, Abbas, M: Fixed point results of cyclic contractions in product spaces. Carpath. J. Math. 31(1) (2015, in press)

20. Luong, NV, Thuan, NX: Some fixed point theorems of Prešić-Ćirić type. Acta Univ. Apulensis 30, 237-249 (2012)

21. Nadler, SB Jr.: Multi-valued contraction mappings. Pac. J. Math. 30, 475-488 (1969)

22. Shukla, S, Sen, R, Radenović, S: Set-valued Prešić type contraction in metric spaces. An. Ştiinţ. Univ. 'Al.I. Cuza' laşi, Mat. (2014, in press)

23. Ran, ACM, Reurings, MCB: A fixed point theorem in partially ordered sets and some application to matrix equations. Proc. Am. Math. Soc. 132, 1435-1443 (2004)

24. Jachymski, J: The contraction principle for mappings on a metric space with a graph. Proc. Am. Math. Soc. 136 1359-1373 (2008)

25. Haghi, RH, Rezapour, S, Shahzad, N: Fixed points of G-type quasi-contractions on graphs. Abstr. Appl. Anal. 2013, Article ID 167530 (2013)

26. Aleomraninejad, SMA, Rezapour, S, Shahzad, N: Some fixed point results on a metric space with a graph. Topol. Appl. $159,659-663(2012)$

27. Samreen, M, Kamran, T, Shahzad, N: Some fixed point theorems in b-metric space endowed with graph. Abstr. Appl. Anal. 2013, Article ID 967132 (2013)

28. Kamran, T, Samreen, M, Shahzad, N: Probabilistic G-contractions. Fixed Point Theory Appl. 2013, 223 (2013)

29. Kirk, WA, Shahzad, N: Fixed Point Theory in Distance Spaces. Springer, New York (2014)

30. Nicolae, A, O'Regan, D, Petrusel, A: Fixed point theorems for singlevalued and multivalued generalized contractions in metric spaces endowed with a graph. Georgian Math. J. 18, 307-327 (2011)

31. Chifu, C, Petrusel, A: Ciric-type delta-contractions in metric spaces endowed with a graph. J. Inequal. Appl. 2014, 77 (2014)

32. Eshaghi Gordji, M, Pirbavafa, S, Ramezani, M, Park, C, Shin, DY: Prešić-Kannan-Rus fixed point theorem on partially order metric spaces. Fixed Point Theory 15(2), 463-474 (2014)

33. Johnsonbaugh, R: Discrete Mathematics. Prentice Hall, New York (1997)

34. Edelstein, M: An extension of Banach's contraction principle. Proc. Am. Math. Soc. 12, 7-10 (1961) 